\title{
Health Status Deterioration in Patients with Chronic Obstructive Pulmonary Disease
}

\author{
SAlly SPENCER, PETER M. A. CALVERLEY, P. SHERWOOD BURGE, and PAUL W. JONES on behalf of the \\ ISOLDE Study Group
}

Department of Physiological Medicine, St George's Hospital Medical School, London, United Kingdom

This study examined health status decline in patients with chronic obstructive pulmonary disease (COPD). Data are from the Inhaled Steroids in Obstructive Lung Disease (ISOLDE) trial. After an 8-wk run-in, 751 patients ( 566 male), mean age $64 \mathrm{yr}$, were randomized to receive fluticasone propionate (FP) $500 \mu \mathrm{g}$ twice daily (376 patients) or placebo ( 375 patients). Mean baseline postbronchodilator $\mathrm{FEV}_{1}$ was $50 \pm 15 \%$ predicted. Patients completed the St George's Respiratory Questionnaire (SGRQ) and the Short-Form 36 (SF-36) at baseline and every 6 mo for $3 \mathrm{yr}$. FEV F $_{1}$ and smoking status were assessed at baseline and at 3-mo intervals. A total of 387 (212 FP) patients completed the trial. All SGRQ components ( $p=0.03$ to 0.004$)$ and Physical Function, Mental Health, Energy/ Vitality, and Physical Role Limitation scales of the SF-36 ( $p=0.05$ to 0.005 ) deteriorated faster in the placebo group. FEV F $_{1}$ and SGRQ scores correlated at baseline values $(r=-0.25, p<0.0001)$, as did change in $\mathrm{FEV}_{1}$ and change in SGRQ $(\Delta r=-0.24, p<0.0001)$. At baseline values smokers had worse SGRQ Total, Symptoms, and Impacts scores than ex-smokers. This difference was maintained throughout the study. Smoking status did not influence the rate of decline in health status. The SGRQ Total scores of FP-treated patients took $59 \%$ longer than placebo to deteriorate by a clinically significant amount. We conclude that health status decline in moderate to severe COPD can be reduced by high-dose fluticasone.

An accelerated decline of $\mathrm{FEV}_{1}$ is well-documented in patients with chronic obstructive pulmonary disease (COPD). Although $\mathrm{FEV}_{1}$ is a useful measure for diagnosis and prognosis, it is neither a good predictor of the symptomatic response to treatment in COPD (1) nor of the likelihood of hospitalization (2). This has led to the use of generic and disease-specific questionnaires to measure the impact of COPD on health status. Crosssectional studies have shown that patient populations with a lower $\mathrm{FEV}_{1}$ have worse health-related quality of life (3). However, to our knowledge there are no data concerning the rate of change in health status within patients over time.

We hypothesized that decline in health status would be detectable in a population of patients in whom COPD was the principal pathology and that this would be amenable to treatment. All components of the disease-specific St George's Respiratory Questionnaire (SGRQ) have shown sensitivity to impaired health in COPD (4). The physical function components of the generic Short-Form 36 (SF-36) have demonstrated sensitivity to change in COPD and to treatment with fluticasone in severe asthma $(5,6)$ so we expected these components of the SGRQ and SF-36 to show the greatest sensitivity to change in our study. Because it is known that smoking has an effect on rate of decline in $\mathrm{FEV}_{1}$, we also hypothesized that smoking status would influence health status and its rate

(Received in original form May 2, 2000 and in revised form August 16, 2000) Supported by a grant from Glaxo Wellcome Research \& Development, UK. Correspondence and requests for reprints should be addressed to Sally Spencer, Department of Physiological Medicine, St George's Hospital Medical School, Cranmer Terrace, London SW17 ORE, UK. E-mail: sspencer@sghms.ac.uk

Am J Respir Crit Care Med Vol 163. pp 122-128, 2001

Internet address: www.atsjournals.org of deterioration. We used data collected as part of the Inhaled Steroids in Obstructive Lung Disease (ISOLDE) study of fluticasone in COPD. The intention-to-treat analyses of the principal outcomes of this trial are reported elsewhere (7). For this analysis, health status was measured using a generic instrument, the SF-36 and a disease-specific instrument, the SGRQ.

\section{METHODS}

\section{Patients}

Full details of the study methodology, patient selection, and spirometric procedures are presented elsewhere (7). Seven hundred fifty-one patients (560 men) with a diagnosis of COPD consistent with American Thoracic Society (ATS) recommendations (8) were recruited from 18 centers throughout the United Kingdom. All were current or exsmokers age 40 to $75 \mathrm{yr}$ with a postbronchodilator $\mathrm{FEV}_{1}$ of at least $0.8 \mathrm{~L}$, but less than $85 \%$ predicted. The $\mathrm{FEV}_{1} / \mathrm{FVC}$ ratio was less than $70 \%$. Exclusion criteria were: an $\mathrm{FEV}_{1}$ response to $400 \mu \mathrm{g}$ salbutamol of $>10 \%$ predicted; use of $\beta$-adrenergic blockers; use of nontrial inhaled corticosteroids or regular oral corticosteroid; a life expectancy of less than $5 \mathrm{yr}$ from non-COPD diseases. At baseline values, postbronchodilator $\mathrm{FEV}_{1}$ expressed as percent of predicted normal was $50 \pm 15$ (SD)\%. The mean $\mathrm{FEV}_{1}$ response to salbutamol was $4.4 \%$ predicted $(0.13 \mathrm{~L})$. During the study, patients requiring long-term treatment with oral corticosteroids or more than two short courses of treatment in any 3-mo period were withdrawn. Ethical committee approval was obtained in each participating center and informed consent was obtained before entry to the study.

\section{Health Status Measurement}

General Health Status was assessed using the SF-36 (9), a generic measure of health impairment (10). It is a self-completed questionnaire containing 36 questions covering eight health concepts: Physical Function, Physical Role Limitation, Mental Role Limitation, Social Function, Mental Health, Pain, Energy/Vitality, and Health Perception. Two summary components (Physical and Mental) can also be calculated by differentially weighting the scales. The SF-36 scales are scored as a percentage of impairment with zero representing worst health and 100 indicating best health. With this scale, a decrease in score indicates worsening general health. Its reliability is extensively documented (11).

The SGRQ (12) is a supervised, self-administered measure designed specifically for use in airways disease. It is a 50-item survey from which are calculated the Total and three component scores: Symptoms (distress caused by respiratory symptoms), Activity (physical activities that cause or are limited by breathlessness), and Impacts (social and psychological effects of the disease). The SGRQ is scored from zero to 100 , where zero indicates best health and 100 indicates worst health. A change in score of four units is consistent with a clinically significant change in the patient. This estimate was made in patients with a similar degree of airway obstruction and level of SGRQ score to patients recruited to this study $(4,13)$. An increase in score indicates worsening health status. The SGRQ has been shown to be a valid measure of health impairment in chronic airflow limitation and to respond to change with therapy $(1,12,14)$.

Normative values for SF-36 were taken from the Oxford Healthy Life Survey (15). These were subjects with a mean age of $51 \mathrm{yr}$ (SD 7 yr) who did not report a chronic illness or a medical consultation in the $2 \mathrm{wk}$ before assessment. The SGRQ control scores were obtained from a study of healthy subjects age 17 to 80 with no current or previ- 
ous history of chest disease who were free of viral upper respiratory tract infection. For this comparison, scores from a cohort of this sample in the same age range as ISOLDE patients were used. Their mean age was $60 \mathrm{yr}$ (SD $12 \mathrm{yr}$ ).

\section{Spirometry}

Spirometry was measured according to ATS criteria for acceptability and reproducibility (16) using a computerized rolling-seal spirometer (SensorMedics 2130D, Netherlands). All $\mathrm{FEV}_{1}$ measurements reported here were obtained postbronchodilator.

\section{Protocol}

Patients entered an 8-wk run-in period during which any current inhaled corticosteroid treatment was discontinued. Postbronchodilator spirometry was assessed at 0,4 , and $8 \mathrm{wk}$. Patients were offered $14 \mathrm{~d}$ treatment with oral prednisolone $0.6 \mathrm{mg} / \mathrm{kg} / \mathrm{d}$. They were then randomized to enter a 3-yr double-blind comparison of inhaled fluticasone propionate (FP) $500 \mu \mathrm{g}$ or matched placebo twice daily via metered-dose inhaler and spacer. The SGRQ and SF-36 were completed after the run-in at $8 \mathrm{wk}$ and then at 6 -mo intervals for $3 \mathrm{yr}$. Patients completed questionnaires on the same day that lung function was tested. Both questionnaires were completed under the supervision of clinical staff and according to guidelines in the relevant manuals. Clinical staff received training on questionnaire administration. Questionnaires were monitored for missed items throughout the study and reports sent to each center every 6 mo.

Smoking history was evaluated using the Medical Research Council bronchitis questionnaire at baseline values and self-reported smoking status recorded 3 mo thereafter. This was confirmed by measurement of exhaled carbon monoxide and urinary cotinine. Details of the evaluation of smoking status have been reported previously (7). Patients who continued to smoke throughout the study (38\%) were defined as smokers (Placebo 147, FP 137). Patients who had not smoked during the study (46\%) were defined as ex-smokers (Placebo 172, FP 176), and $16 \%$ were mixed smokers who had either resumed smoking or had quit during the study (Placebo 56, FP 63).

\section{Statistical Analysis}

The purpose of the study was to compute the rate or velocity of decline in health status. The SGRQ and SF-36 scores were calculated using the methods documented in their respective manuals. This included handling of individual missing questionnaire items. Because there were significantly more patients in the placebo group who failed to complete the study than FP-treated patients (7), the rate of decline had to be calculated using a method that took into account this differential drop-out pattern. A random coefficients hierarchical model was used, in which the regression estimates were weighted by the number of observations and the amount of variance contributed by each patient (17). Patients who did not complete the study contributed less weight to the model than those who did complete. Nonlinearity in the slopes was tested by fitting a quadratic term for time. The intentionto-treat population was used for the comparison of FP against placebo. In that analysis, covariates in the model included baseline health status score, smoking status during the study, sex, age at baseline, and study center. These covariates were entered as fixed effects. Slopes were allowed to vary between patients, so time was entered as a random effect. Differences in rate of deterioration between the treatment and placebo groups were tested using a treatment-by-time interaction term. Baseline health status score was used as a covariate in the model, so the rate of decline was estimated using data from the 6-mo assessment onward, to a maximum of 6 assessments per subject. The numbers of patients included in each analysis varied, owing to patient withdrawal or missing data.

The principle hypotheses to be tested were: (1) Health status would decline more slowly in patients treated with FP compared with placebo. (2) Smokers would have worse health status than ex-smokers and their rate of decline in health status decline would be higher than ex-smokers. (3) Baseline $\mathrm{FEV}_{1}$ percentage of predicted would be related to baseline health status but would not predict health status decline. (4) Health status decline and $\mathrm{FEV}_{1}$ decline would be correlated.

\section{RESULTS}

We received over 3,500 completed versions of each questionnaire; less than $4 \%$ of the questionnaires were not returned, i.e., were classified as missing data. The number of SGRQ and SF-36 questionnaires collected at each measurement occasion are shown in Table 1. There were 387 patients remaining in the study at the end of 36 mo.

\section{Baseline Health Status}

All baseline SGRQ scores were higher than scores from the reference population, indicating that these patients had worse health compared with normal subjects (Table 2). Baseline SGRQ Total, Symptoms, and Activity scores were not different between treatment groups (Table 2) although the baseline Impact score was marginally worse in the placebo group compared with FP ( $p=0.03)$. The baseline scores for the physical components of the SF-36 were lower than healthy subject scores (indicating worse health status) and did not differ between FP and placebo groups. In contrast, the baseline SF-36 Mental Health and Mental Summary scores were only slightly lower than those in normal subjects. The baseline score for Mental Health was worse in the placebo group compared with FP $(p=0.04)$.

Worse baseline health status was associated with lower baseline $\mathrm{FEV}_{1}$. All correlations between baseline SGRQ scores and baseline $\mathrm{FEV}_{1}$ percentage of predicted were low, but statistically significant $(\mathrm{r}=-0.12$ to $-0.31, \mathrm{p}<0.01$ to $\mathrm{p}<$ 0.0001). The SF-36 Physical Function, Physical Role Limitation, Physical Summary, Social Function, and Health Perception scores were significantly related to baseline $\mathrm{FEV}_{1}$ values ( $\mathrm{r}=0.15$ to $0.25, \mathrm{p}<0.001$ to $\mathrm{p}<0.0001$ ), again showing that a lower $\mathrm{FEV}_{1}$ was related to worse general health.

\section{Change in Health Status in the First 6 mo}

Over this period, the SGRQ Activity score improved slightly in the FP group and worsened in the placebo group $(\Delta \mathrm{FP}=$ $-0.2 ; \Delta$ Placebo $=+2.7: p=0.03)$. Changes in the other SGRQ scores and the SF-36 scores over the same period were not significant. The change in SGRQ Total score from baseline to $6 \mathrm{mo}$ is shown in Figure 1.

\section{Deterioration in Health Status Over 3 yr}

Health status declined progressively in all components of the SGRQ $(p<0.0001)$ and in all components of the SF-36 ( $<<$ $0.001)$. The rate of deterioration in health status was linear with no significant polynomial component in any score.

The SGRQ Total score worsened by 2.0 units per year in the FP-treated group and by 3.2 units per year in the placebo group (Table 3). FP reduced the rate of deterioration in the Total score by $37 \%$ compared with placebo $(p=0.004)$. The greatest effect of treatment was seen in the Symptoms compo-

TABLE 1

NUMBER OF QUESTIONNAIRES RECEIVED*

\begin{tabular}{lrrr}
\hline & SGRQ & SF-36 & Patients in the Study (FP) \\
\hline Baseline & 736 & 732 & $751(376)$ \\
$6 \mathrm{mo}$ & 608 & 610 & $624(320)$ \\
$12 \mathrm{mo}$ & 534 & 530 & $548(289)$ \\
$18 \mathrm{mo}$ & 490 & 488 & $506(267)$ \\
$24 \mathrm{mo}$ & 449 & 452 & $468(252)$ \\
$30 \mathrm{mo}$ & 412 & 410 & $424(232)$ \\
$36 \mathrm{mo}$ & 368 & 364 & $394(212)$ \\
Total received & 3,597 & 3,586 & \\
Not received (\%) & $122(3.3)$ & $133(3.7)$ & \\
\hline
\end{tabular}

* Unequal numbers are due to patients attending visits but not completing questionnaires. Declining numbers over time are due to patients withdrawing from the study. 
TABLE 2

HEALTHY SUBJECT AND BASELINE ISOLDE PATIENT SCORES

\begin{tabular}{|c|c|c|c|c|}
\hline & $\begin{array}{l}\text { Healthy Subjects } \\
\text { (mean } \pm S D)\end{array}$ & $\begin{array}{c}\text { Placebo } \\
(\text { mean } \pm S D)\end{array}$ & $\begin{array}{c}\mathrm{FP} \\
(\text { mean } \pm S D)\end{array}$ & $\begin{array}{c}\text { Placebo - FP } \\
\text { Difference } \\
(p \text { Value })\end{array}$ \\
\hline \multicolumn{5}{|l|}{ SGRQ } \\
\hline Total & $7 \pm 7$ & $50 \pm 17$ & $48 \pm 18$ & 0.08 \\
\hline Symptoms & $12 \pm 15$ & $67 \pm 22$ & $66 \pm 21$ & 0.5 \\
\hline Activity & $11 \pm 13$ & $62 \pm 21$ & $61 \pm 22$ & 0.4 \\
\hline Impact & $3 \pm 5$ & $38 \pm 19$ & $35 \pm 19$ & 0.03 \\
\hline \multicolumn{5}{|l|}{ SF-36 } \\
\hline Physical function & $91 \pm 13$ & $40 \pm 26$ & $44 \pm 25$ & 0.09 \\
\hline Mental health & $78 \pm 16$ & $70 \pm 18$ & $73 \pm 18$ & 0.04 \\
\hline Energy/Vitality & $66 \pm 18$ & $46 \pm 21$ & $48 \pm 20$ & 0.3 \\
\hline Health perception & $79 \pm 15$ & $40 \pm 21$ & $42 \pm 20$ & 0.1 \\
\hline Physical role limitation & $93 \pm 20$ & $40 \pm 42$ & $44 \pm 41$ & 0.1 \\
\hline Mental role limitation & $89 \pm 26$ & $59 \pm 43$ & $63 \pm 42$ & 0.2 \\
\hline Social function & $94 \pm 14$ & $69 \pm 28$ & $71 \pm 27$ & 0.3 \\
\hline Pain & $88 \pm 16$ & $73 \pm 27$ & $74 \pm 27$ & 0.6 \\
\hline Physical summary & $55 \pm 5$ & $35 \pm 10$ & $36 \pm 10$ & 0.2 \\
\hline Mental summary & $53 \pm 6$ & $49 \pm 11$ & $50 \pm 10$ & 0.06 \\
\hline
\end{tabular}

nent of the SGRQ in which FP significantly reduced the rate of deterioration in scores by $56 \%$. FP reduced the rate of decline in Activity scores by $39 \%$ and in Impacts scores by $33 \%$ compared with placebo (Figure 2). The SGRQ Total score in the placebo group took $1.26 \mathrm{yr}$ (approximately $15 \mathrm{mo}$ ) to deteriorate by the minimal clinically important difference of 4 units, compared with $2.00 \mathrm{yr}$ in the FP group (Figure 2).

Fluticasone significantly reduced the rate of deterioration in SF-36 scores for Physical Function, Energy/Vitality, and Physical Role Limitation (Table 4); however, the Physical Summary score which summarizes all SF-36 components related to physical impairment was not influenced by treatment (Figure 2). Of the psychosocial components, only the deterioration in Mental Health scores was significantly different between treatments. In the FP-treated patients, this score did not change, but the placebo-treated patients showed a significant deterioration. The deterioration in Mental Role Limitation, General Health Perception, Social Function, Pain, and Mental Summary scores was not significantly different between FP and placebo. No minimal clinically important difference value has been established for the SF-36 in patients with COPD, so the clinical significance of these scores cannot be assessed.

\section{Influence of Baseline $\mathrm{FEV}_{1}$ on Health Status Deterioration}

A lower $\mathrm{FEV}_{1}$ at baseline was associated with a more rapid rate of decline in SGRQ Impacts scores $(p=0.008)$. There

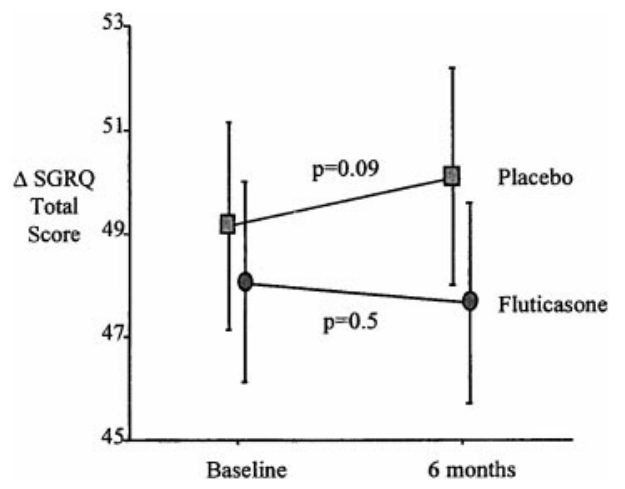

Figure 1. Change in SGRQ Total score over the first 6 mo of treatment for placebo-treated patients (squares) and FP-treated patients (circles). Higher SGRQ score indicates worse health. Bars represent 95\% confidence intervals. were no significant differences in this relationship between treatment groups $(\mathrm{p}>0.05)$. There was no significant relationship between baseline $\mathrm{FEV}_{1}$ and the rate of decline in the SGRQ Total, Symptoms, or Activity scores $(\mathrm{p}>0.05)$. There was no significant relationship between baseline $\mathrm{FEV}_{1}$ and the rates of decline in any of the SF-36 scales ( $p>0.05)$.

\section{Influence of Smoking on Baseline Health Status}

Analysis was restricted to those who either smoked for the duration of the study or who remained ex-smokers throughout. Smokers had worse baseline SGRQ scores than ex-smokers: Total (difference $=3.6, \mathrm{p}=0.04)$, Symptoms $($ difference $=$ 9.7, $\mathrm{p}<0.0001$ ), and Impacts (difference $=4.7, \mathrm{p}=0.01)$. The baseline Activity scores did not differ by smoking status. Smokers had worse baseline SF-36 scores than ex-smokers for Mental Health (difference $=-5.1, \mathrm{p}=0.003$ ), Mental Role Limitation (difference $=-10.8, \mathrm{p}=0.007)$, Mental Summary (difference $=-3.6, \mathrm{p}=0.0004)$, Health Perception (difference $=-4.8, p=0.02)$, Energy/Vitality (difference $=-6.1, p=$ 0.002 ), and Pain (difference $=-6.2, \mathrm{p}=0.02$ ). There were no significant differences between the baseline scores of smokers and ex-smokers on any of the remaining scales: Physical Function, Physical Role Limitation, Physical Summary, and Social Function. Multivariate analysis showed that differences in health status between current and ex-smokers were not attributable to differences in $\mathrm{FEV}_{1}$.
TABLE 3

DIFFERENCE IN SGRQ RATES OF DETERIORATION BETWEEN FP AND PLACEBO*

\begin{tabular}{|c|c|c|c|c|c|}
\hline & $\mathrm{n}$ & $\begin{array}{c}\text { FP } \\
\text { (Units/yr) } \\
\text { (SE) }\end{array}$ & $\begin{array}{c}\text { Placebo } \\
\text { (Units/yr) } \\
\text { (SE) }\end{array}$ & $\begin{array}{c}\text { FP-Placebo } \\
\text { Difference } \\
(95 \% \mathrm{CI})\end{array}$ & $p$ Value \\
\hline Total & 600 & $\begin{array}{c}2.00 \\
(0.29)\end{array}$ & $\begin{array}{c}3.17 \\
(0.31)\end{array}$ & $\begin{array}{c}-1.17 \\
(-0.39,-1.95)\end{array}$ & 0.004 \\
\hline Symptoms & 600 & $1.02 \quad(0.37)$ & $\begin{array}{l}2.33 \\
(0.40)\end{array}$ & $\begin{array}{c}-1.31 \\
(-0.37,-2.25)\end{array}$ & 0.007 \\
\hline Activity & 600 & $2.10 \quad(0.35)$ & $\begin{array}{c}3.47 \\
(0.38)\end{array}$ & $\begin{array}{c}-1.37 \\
(-0.45,-2.29)\end{array}$ & 0.004 \\
\hline Impacts & 600 & $2.17 \quad(0.36)$ & $\begin{array}{c}3.24 \\
(0.38)\end{array}$ & $\begin{array}{c}-1.07 \\
(-0.11,-2.03)\end{array}$ & 0.03 \\
\hline
\end{tabular}

* The higher the slope, the greater the rate of declilne of health status. 

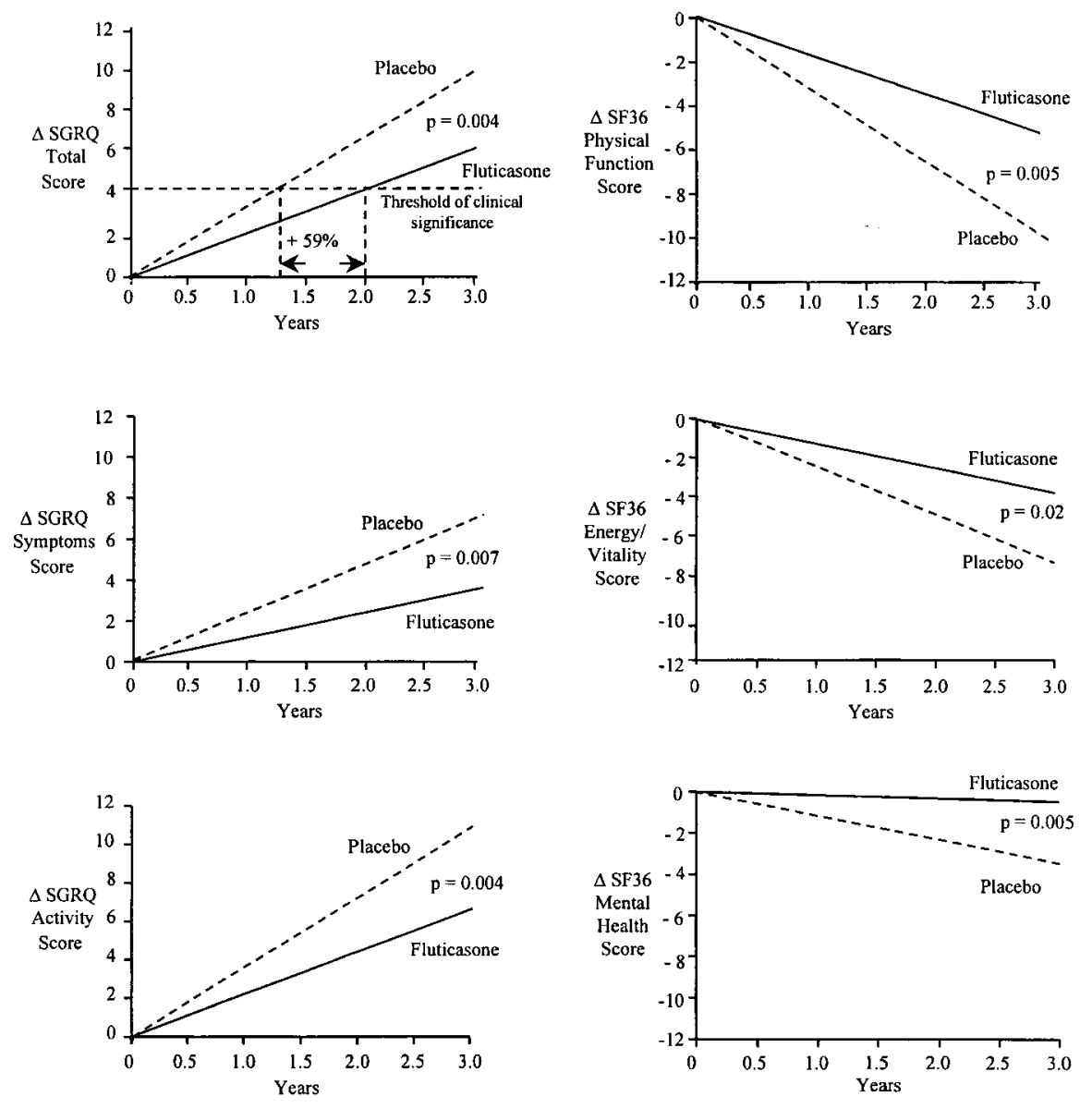

Figure 2. Slope of deterioration in health status calculated using estimates from a random coefficients hierarchical model. The regression estimates are weighted by the number of observations and the variance contributed by each patient. A higher SGRQ score or a lower SF-36 score indicates worse health status. All SGRQ component scores are shown, together with selected SF-36 scores.
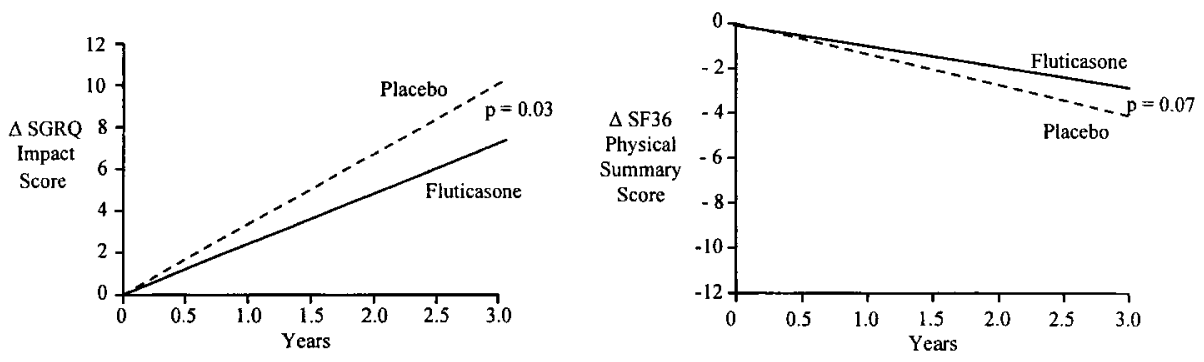

\section{Influence of Smoking on Health Status Deterioration}

The differences between smokers and ex-smokers observed in the SGRQ components at baseline values were maintained throughout the study. The mean difference in score between smokers and nonsmokers over three yr was: Total $4.3(\mathrm{p}=$ 0.002); Symptoms 5.4 ( $\mathrm{p}=0.0001)$; Impacts $5.6(\mathrm{p}=0.0005)$. The Activity score remained not different between the two groups $(\mathrm{p}=0.8)$. Smoking status had no effect on the rate of deterioration in SGRQ score, i.e., smokers and ex-smokers declined in parallel (Figure 3). Smoking had no influence on the effect of FP on decline in health status.

Differences in the three SF-36 mental health scores between smokers and ex-smokers at baseline were maintained throughout the study $(\mathrm{p}<0.05)$. On average Social Function scores were worse in smokers during the study period, although there was no significant difference at baseline. There were no differences in the rate of deterioration of SF-36 scores between smokers and ex-smokers, neither did smoking status influence the effect of FP on change in health status.
Relationship between Health Status Decline and the Rate of Decline in $\mathrm{FEV}_{\mathbf{1}}$

Details of changes in $\mathrm{FEV}_{1}$ during the study have been reported elsewhere (7). The current analysis showed that there was a small but significant linear relationship between the rate of decline in SGRQ scores and rate of decline in $\mathrm{FEV}_{1}$ : Total = $\mathrm{R}-0.24, \mathrm{p}<0.0001$; Symptoms $=\mathrm{R}-0.14, \mathrm{p}=0.0005$; Activity $=\mathrm{R}-0.25, \mathrm{p}<0.0001$; Impacts $=\mathrm{R}-0.18, \mathrm{p}<0.0001$. There was a similar relationship between the rate of decline in the physical components of the SF-36 and the rate of decline in $\mathrm{FEV}_{1}$ : Physical Function $=\mathrm{R} 0.22, \mathrm{p}<0.0001$; Physical Role Limitation $=\mathrm{R} 0.12, \mathrm{p}=0.005$; Energy $/$ Vitality $=\mathrm{R}$ $0.15, \mathrm{p}=0.0003$; Health Perception $=\mathrm{R} 0.15, \mathrm{p}=0.0003$; Physical Summary $=\mathrm{R} 0.21, \mathrm{p}<0.0001$. Rates of decline in the Pain scale and the psychosocial components of the SF-36 (Mental Health, Mental Role Limitation, Social Function, and Mental Summary) were unrelated to the rate of decline in $\mathrm{FEV}_{1}(\mathrm{p}>0.05)$. The relationship between health status decline and $\mathrm{FEV}_{1}$ decline did not differ between treatments $(\mathrm{p}>$ 
TABLE 4

DIFFERENCE IN SF-36 RATES OF DETERIORATION BETWEEN FP AND PLACEBO*

\begin{tabular}{|c|c|c|c|c|c|}
\hline & $\mathrm{n}$ & $\begin{array}{c}\text { FP } \\
\text { (Units/yr) } \\
\text { (SE) }\end{array}$ & $\begin{array}{l}\text { Placebo } \\
\text { (Units/yr) } \\
\text { (SE) }\end{array}$ & $\begin{array}{c}\mathrm{FP}=\text { Placebo } \\
\text { Difference } \\
(95 \% \mathrm{Cl})\end{array}$ & $\mathrm{p}$ Value \\
\hline Physical function & 592 & $\begin{array}{r}-1.73 \\
(0.43)\end{array}$ & $\begin{array}{r}-3.33 \\
(0.46)\end{array}$ & $\begin{array}{c}1.60 \\
(0.50,2.70)\end{array}$ & 0.005 \\
\hline Mental health & 596 & $\begin{array}{c}0.02 \\
(0.34)\end{array}$ & $\begin{array}{c}-1.13 \\
(0.35)\end{array}$ & $\begin{array}{c}1.15 \\
(0.35,1.95)\end{array}$ & 0.005 \\
\hline Energy/vitality & 596 & $\begin{array}{c}-1.20 \\
(0.38)\end{array}$ & $\begin{array}{c}-2.38 \\
(0.41)\end{array}$ & $\begin{array}{c}1.18 \\
(0.24,2.12)\end{array}$ & 0.02 \\
\hline Physical role limitation & 595 & $\begin{array}{r}-2.96 \\
(0.87)\end{array}$ & $\begin{array}{c}-4.97 \\
(0.92)\end{array}$ & $\begin{array}{c}2.01 \\
(0.03,3.99)\end{array}$ & 0.05 \\
\hline Social function & 582 & $\begin{array}{c}-2.55 \\
(0.62)\end{array}$ & $\begin{array}{c}-4.03 \\
(0.67)\end{array}$ & $\begin{array}{c}1.48 \\
(-0.30,2.99)\end{array}$ & 0.06 \\
\hline Pain & 591 & $\begin{array}{r}-1.39 \\
(0.57)\end{array}$ & $\begin{array}{r}-2.28 \\
(0.61)\end{array}$ & $\begin{array}{c}0.89 \\
(-0.50,2.28)\end{array}$ & 0.2 \\
\hline Mental role limitation & 599 & $\begin{array}{c}-4.51 \\
(0.97)\end{array}$ & $\begin{array}{c}-5.56 \\
(1.04)\end{array}$ & $\begin{array}{c}1.05 \\
(-1.24,3.34)\end{array}$ & 0.4 \\
\hline Health perception & 568 & $\begin{array}{r}-2.33 \\
(0.37)\end{array}$ & $\begin{array}{c}-2.77 \\
(0.40)\end{array}$ & $\begin{array}{c}0.44 \\
(-0.52,1.40)\end{array}$ & 0.4 \\
\hline Physical summary & 547 & $\begin{array}{c}-0.86 \\
(0.19)\end{array}$ & $\begin{array}{c}-1.31 \\
(0.20)\end{array}$ & $\begin{array}{c}0.44 \\
(-0.03,0.91)\end{array}$ & 0.07 \\
\hline Mental summary & 547 & $\begin{array}{c}-0.66 \\
(0.23)\end{array}$ & $\begin{array}{c}-1.14 \\
(0.24)\end{array}$ & $\begin{array}{c}0.48 \\
(-0.07,1.03)\end{array}$ & 0.09 \\
\hline
\end{tabular}

* The more negative the slope, the greater the rate of decline of health status.

$0.01)$ or by smoking status $(p>0.01)$ on any of the components of the SGRQ or the SF-36.

\section{DISCUSSION}

We have found clear evidence of a measurable and progressive deterioration in the health of this population of patients with COPD. This was present in all domains of health impairment: symptoms, physical activity, and psychosocial function, whether measured using the disease-specific SGRQ or the generic SF-36. Previous studies have measured change in health status by comparing baseline to endpoint values $(1,12)$ but few have assessed change using more than two measurement points. Other trials have measured health status on multiple occasions but have design limitations such as small sample size (18), sole use of a generic measure (19), or use of a relatively untested generic measure on a male-only population (5). This is the first published study to document health status decline prospectively in a large and well-characterized population.

Particular care was taken to establish a cohort of stable patients with COPD without substantial comorbidity that might confound the health status assessments. These patients appear to be similar to other populations of patients with COPD because the mean value for $\mathrm{FEV}_{1}$ and mean SGRQ Total score measured at baseline in this study lie on a previously reported regression between mean values for these two measures found in other studies (20). The baseline health status scores for the SGRQ showed substantial differences from scores in diseasefree subjects. Most of the SF-36 scores, with the exception of Mental Health, were also significantly worse than in the control subjects.

The rate of deterioration in health status was reduced by inhaled fluticasone. This effect was seen in all domains of health measured using the SGRQ, and the SF-36 Physical Function, Physical Role Limitation, Energy/Vitality, and Mental Health scales. As has been reported previously in patients with COPD (1), the disease-specific questionnaire was more sensitive to therapeutic intervention than the generic instrument. Mechanisms responsible for the beneficial effect of in-

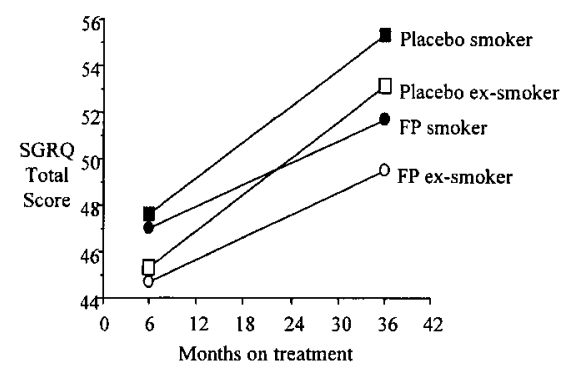

Figure 3. Deterioration in SGRQ score in smokers and ex-smokers (see Figure 2 for method of calculating slopes). Smokers had higher SGRQ scores, i.e., worse health, at baseline. The rate of decline in smokers and ex-smokers was parallel in both treatment arms. Within each treatment group, smokers and ex-smokers declined at the same rate, i.e., smoking status did not influence the treatment effect.

haled steroids have yet to be identified. Our data suggest that it is an effect that accumulates over time because the difference in health of placebo- and fluticasone-treated patients widened progressively. One possible mechanism is a link with exacerbations. In this study, exacerbations that required treatment with oral steroids or antibiotics were reduced by fluticasone (7) and patients with a high number of exacerbations have been reported to have worse SGRQ scores than those with few (21). In support of this hypothesis is the observation that fluticasone exerted its greatest influence on the rate of decline in the Symptoms component of the SGRQ. This addresses the impact of the frequency of episodes of wheeze, cough, and sputum. In terms of consistency across health domains, the drug had greatest influence on deterioration in physical aspects of health rather than psychosocial functions. This observation leads us to speculate that each exacerbation may lead to a step reduction in physical activity and function that may not be fully reversible. By reducing the number of exacerbations, fluticasone would improve symptoms and reduce the rate at which physical function deteriorates.

Could drug-related euphoria account for the effect of fluticasone? Clinical observations of nonspecific improvements in mood with high doses of oral corticosteroids led to their use in palliative care, but well-controlled trials have not been reported. Most reports of the psychological effects of oral corticosteroids have been concerned with major psychoses in patients receiving medium-high dose treatment (greater than prednisolone $40 \mathrm{mg} / \mathrm{d}$ or equivalent). There has been only one study of possible euphoriant effects of corticosteroids in patients with COPD (22). In that study, patients given oral prednisolone $30 \mathrm{mg} / \mathrm{d}$ showed a very small improvement in anxiety and depression after $1 \mathrm{wk}$ of therapy, but those changes had largely reversed after $2 \mathrm{wk}$ despite continued steroid treatment. Oral corticosteroids were given for $2 \mathrm{wk}$ at the start of our study, but this cannot explain the health status benefit of fluticasone because both placebo- and fluticasone-treated patients received prednisolone. It is unlikely that there was any carry-over effect of that treatment, because we found no evidence of improved psychosocial function in either treatment group over the first 6 mo of therapy. Finally, it should be noted also that effects of fluticasone were most consistent in the physical domains of health impairment, rather than the psychological components. We conclude that there is no evidence of a "euphoriant" effect of inhaled steroids in COPD.

Current smokers had worse health at baseline than exsmokers, a difference that was not attributable to differences in $\mathrm{FEV}_{1}$. Reasons for this association are not clear from this study, but it is noteworthy that the SGRQ Symptoms and 
Impact scores and the SF-36 psychosocial scales were most affected by smoking status. In contrast, neither the physically oriented scales of the SF-36 nor the physical Activity component of the SGRQ were related to smoking. Health status declined at the same rate in smokers and ex-smokers, the difference between them neither widening nor narrowing significantly. This is in contrast to the effect of smoking on decline in FEV $_{1}(7)$. The effect of fluticasone on the rate of deterioration in health appeared to be the same in smokers and ex-smokers. To explain these observations, two hypotheses appear equally plausible: either patients with poor psychosocial status are less able to quit smoking, or smoking makes these aspects of health worse. There were too few patients who restarted or stopped smoking during the study to test these hypotheses.

At baseline values there was a weak correlation between $\mathrm{FEV}_{1}$ and SGRQ score. This was expected because weak correlations between health status and spirometry have been reported previously $(3,12,23)$. In contrast, correlations between health status and other physiological measures such as exercise performance and arterial $\mathrm{PO}_{2}$ may be stronger $(12,23,24)$. An association between worse baseline $\mathrm{FEV}_{1}$ and subsequent rate of decline of SGRQ Impacts score was an unexpected finding. If confirmed in further studies it may suggest that health status decline accelerates with worsening lung function. Because cross-sectional studies (i.e., between patients) had shown a significant correlation between $\mathrm{FEV}_{1}$ and health status (3), the weak but significant correlation between decline in $\mathrm{FEV}_{1}$ and decline in health status was expected. It is noteworthy that correlations between $\mathrm{FEV}_{1}$ and SGRQ score were of similar strength whether between or within patients.

The clinical significance of the changes produced by our interventions can be interpreted only for the SGRQ score because there is no defined clinically significant change score for the summary components of the SF-36 when used in COPD. The placebo-treated patients reached a clinically significant level of deterioration of 4 units in SGRQ Total score $(4,13)$ every 15 mo. Those receiving fluticasone took 24 mo to decline by the same amount. To set these values into a clinical context, it is necessary to "back-translate" a 4-unit change in SGRQ score into patient scenarios. Two examples of what a 4-unit deterioration may mean to a patient are illustrated here. Each should be seen as being a comparison with a previous assessment. Example 1: The patient now has to walk slower than others of his or her age, has become breathless on getting washed or dressed, and is now breathless on bending over. Example 2: The patient now takes a long time to wash or dress, can no longer walk up stairs without stopping, and cannot now go out for entertainment. To achieve a 4-unit deterioration in SGRQ, the patient must have experienced all three components described in each scenario. It is quite clear that the degree of deterioration illustrated by these two examples would be quite significant in a patient's life. This degree of worsening occurs every $15 \mathrm{mo}$, but fluticasone increases the time it takes for this to occur by $58 \%$. This appears to be a worthwhile therapeutic benefit.

This study has shown that it possible to measure the rate of decline of health status in COPD and to detect worthwhile treatment benefits; however, there are some limitations to the generalizability of the results. First, the entry criteria excluded patients with COPD with a high level of bronchodilator reversibility, because we were anxious to exclude patients who may be considered to be asthmatic. We believe that this entry criterion was unlikely to have biased the study in favor of a large treatment effect, however. Second, many patients who entered the study had survived withdrawal from inhaled steroids to enter the trial. A previous publication reported that such patients had a higher number of exacerbations during the run-in phase of the study than patients who were not withdrawn from (25). As a result, possible "steroid-dependent" patients did not enter the study and may have biased the result toward an underestimate of the "true" steroid effect to be obtained in COPD. Third, post hoc subgroup analysis (not reported here) showed that patients who withdrew from the study had a significantly higher rate of decline of health status than those who completed the 3-yr treatment period. These patients also showed the greatest effect of FP compared with placebo. The hierarchical statistical model used for this analysis placed less weight on data from these patients because they contributed fewer observations than patients who completed the study. Overall, it appears that the patient entry criteria and method of analysis may have led to a conservative estimate of the effect of FP in patients with COPD.

This study has a number of implications for the treatment of COPD and for clinical trials in this field. We have shown that the health of patients with COPD patients treated with bronchodilator therapy alone deteriorates by a clinically significant amount every 15 mo. This deterioration can be detected using both disease-specific and generic measurements; however, the former gave more consistent results and may be more sensitive to change. Maintenance therapy with FP reduced the rate at which deterioration progressed. This benefit, best seen in the physical components of health, required time to accumulate. Continuing smokers had worse health than did patients who had stopped, but it is not clear whether smoking cessation is the cause for the better level of health. The ISOLDE study has demonstrated that measuring health status is valuable in long-term trials designed to establish whether therapy alters progression of COPD.

\section{References}

1. Jones PW Bosh TK. Quality of life changes in COPD patients treated with salmeterol. Am J Respir Crit Care Med 1997;155:1283-1289.

2. Osman LM, Godden DJ, Friend JAR, Legge JS, Douglas JG. Quality of life and hospital re-admission in COPD. Eur Respir J 1996;9(Suppl 23):143s.

3. Ferrer M, Alonso J, Prieto L, Plaza V, Mono E, Marrades R, Aguar MC, Khalaf A, Anto JM. Validity and reliability of the St George's Respiratory Questionnaire after adaptation to a different language and culture: the Spanish example. Eur Respir J 1996;9:1160-1166.

4. Jones PW, Quirk FH, Baveystock CM. The St George's Respiratory Questionnaire. Respir Med 1991;85(Suppl B):25-31.

5. Mahler DA, Tomliinson D, Olmstead EM, Tosteson ANA, and O'Connor GT. Changes in dyspnea, health status, and lung function in chronic airways disease. Am J Respir Crit Care Med 1995;151:61-65.

6. Noonan M, Chervinsky P, Busse WW, Weisberg SC, Pinnas J, de Boisblanc BP, Boltansky H, Pearlman D, Repsher L, Kellerman D. Fluticasone propionate reduces oral prednisone use while it improves asthma control and quality of life. Am J Respir Crit Care Med 1995;152:1467-1473.

7. Burge PS, Calverley PMA, Jones PW, Spencer S, Anderson JA, Maslen TK. Randomised, double-blind, placebo controlled study of fluticasone propionate in patients with moderate to severe chronic obstructive pulmonary disease; the ISOLDE trial. BMJ 2000;320:1297-1303.

8. American Thoracic Society. Standards for the diagnosis and care of patients with chronic obstructive pulmonary disease (COPD) and asthma. Am Rev Respir Dis 1987;136:225-241.

9. Ware J Sherbourne C. The MOS 36-Item Short-Form Health Survey (SF-36): 1. Conceptual framework and item selection. Med Care 1992; 30:473-481.

10. Bowling A. Measuring health: a review of quality of life measurement scales. Buckingham: Open University Press; 1995.

11. Brazier J, Harper R, Jones N, O'Cathain A, Thomas K, Usherwood T, Westlake L. Validating the SF-36 health survey questionniare: new outcome measure for primary care. BMJ 1992;305:160-164.

12. Jones PW, Quirk FH, Baveystock CM, Littlejohns P. A self-complete measure for chronic airflow limitation-the St George's Respiratory Questionnaire. Am Rev Respir Dis 1992;145:1321-1327. 
13. Jones PW, Lasserson D. Relationship between change in St George's Respiratory Questionnaire score and patient's perception of treatment efficacy after one year of therapy with nedocromil sodium [abstract]. Am Rev Respir Crit Care Med 1994;149:A211.

14. Meecham-Jones DJ, Paul EA, Jones PW, Wedzicha JA. Nasal pressure support ventilation plus oxygen compared with oxygen therapy alone in hypercapnic COPD. Am J Respir Crit Care Med 1995;152:538-544.

15. Jenkinson C, Coulter A, Wright L. Short form 36 (SF 36) health survey questionnaire: normative data for adults of working age. BMJ 1993; 306:1437-1440.

16. American Thoracic Society. Standardization of spirometry. Am J Respir Crit Care Med 1995;152:1107-1136.

17. Goldstein H. Multilevel statistical models, 2nd ed. London: Edward Arnold; 1995.

18. van Schayck CP, Dompeling E, Rutten MP, Folgering H, van den Boom $\mathrm{G}$, van Weel C. The influence of an inhaled steroid on patients with asthma or COPD. Chest 1995;107:1199-205.

19. Intermittent Positive Pressure Trial Group. Intermittent positive pressure breathing therapy of chronic obstructive pulmonary disease. Ann Intern Med 1983;99:612-620.
20. Jones PW. Quality of life, health economics and asthma. Eur Respir Rev 1995;5:279-283.

21. Seemungal TAR, Donaldson GC, Paul EA, Bestall JC, Jeffries DJ, Wedzicha JA. Effect of exacerbation on quality of life in patients with chronic obstructive pulmonary disease. Am J Respir Crit Care Med 1998;157:1418-1422.

22. Swinburn CR, Wakefield JM, Newman SP, Jones PW. Evidence of prednisolone induced mood change ('steroid euphoria') in patients with chronic obstructive airways disease. Br J Clin Pharmac 1988;26:709-713.

23. Jones PW, Baveystock CM, Littlejohns P. Relationships between general health measured with the Sickness Impact Profile and respiratory symptoms, physiological measures and mood in patients with chronic airflow limitation. Am Rev Respir Dis 1989;140:1538-1543.

24. Okubadeyo AA, Jones PW, Wedzicha JA. Quality of life in patients with chronic obstructive pulmonary disease and severe hypoxaemia. Thorax 1996;51:44-47.

25. Jarad NA, Wedzicha JA, Burge PS, Calverley PMA, and for the Isolde group. An observational study of inhaled corticosteroid withdrawal in stable chronic obstructive pulmonary disease. Respir Med 1999;93:161166. 